\title{
Understanding the adjustment of children with special needs: A family systems perspective
}

Chun Bun Lam and Kevin Kien Hoa Chung,

The Department of Early Childhood Education, The Education University of Hong Kong

\section{Introduction}

According to a family system perspective, children are shaped by multiple, interrelated socialization forces, nested within, for example, the parents, the parent-child relationship, the inter-parental relationship, and the larger family (Parfitt et al., 2014). The unique contributions of different family subsystems to the adjustment of children, especially those with special needs (SNs), are rarely tested empirically, however (Algood et al., 2013). Existing research also tends to focus on the roles of mothers in European American and European families. The roles of fathers in Asian families continue to be under explored (Li et al., 2019)

Using cross-sectional data collected from Chinese families with kindergarten-aged children with SNs, the present study examined whether paternal and maternal depressive symptoms, father- and mother-child conflict, inter-parental conflict, and family economic pressure were independently linked to child internalizing symptoms and externalizing behaviors.

\section{Participants and procedures}

Participants were 98 fathers and 98 mothers from 98 families with children with SNs, recruited from 20 integrated kindergarten-cum-child care centres in Hong Kong, China. These centres aimed to educate typically developing children and children with SNs in a more integrated setting. Major types of SNs included global developmental delay, speech and language developmental delay, motor developmental delay, autistic spectrum disorder, and attention deficit and hyperactivity disorder.

Fathers and mothers provided demographic information, including child gender and age, and independently completed questionnaires on family relationships and child adjustment. The mean age of children was 61.89 months (SD $=10.51)$. Seventy-four percent of these children were boys ( $n=71)$. Fathers' and mothers' reports of inter-parental conflict, family economic pressure, and child adjustment problems were averaged, as they were highly correlated ( $r s>.60)$.

\section{Results}

Multiple regression analyses indicated that, controlling for child gender and age, maternal depressive symptoms $(\beta=.30 ; t=$ $2.42 ; p<.05)$ and family economic pressure $(\beta=.29 ; t=2.69 ; p<.01)$ were uniquely and positively linked to child internalizing symptoms. Moreover, father-child conflict $(\beta=$ .35; $t=3.17 ; p<.01$ ) and motherchild conflict $(\beta=.24 ; t=2.19 ; p<$ .05) were uniquely and positively linked to child externalizing symptoms (see Table below).
香港教育大學

The Education University of Hong Kong

\begin{tabular}{|l|l|c|c|c|c|}
\hline \multicolumn{2}{|c}{} & \multicolumn{2}{c}{ Internalizing } & \multicolumn{3}{c|}{ Externalizing } \\
\hline Steps & \multicolumn{1}{|c|}{ Variables } & $\beta$ & $t$ values & $\beta$ & t values \\
\hline 1 & Child gender & 0.12 & 1.16 & 0.08 & .78 \\
\hline & Child age & -.06 & -0.55 & 0.10 & 1.00 \\
\hline 2 & Father depression & -0.06 & -0.43 & -0.07 & -0.55 \\
\hline \multirow{2}{*}{} & Mother depression & 0.30 & $2.42^{*}$ & 0.00 & 0.00 \\
\hline & Father-child conflict & 0.12 & 0.99 & 0.35 & $3.17^{* *}$ \\
\hline & Mother-child conflict & 0.10 & 0.88 & 0.24 & $2.19^{*}$ \\
\hline & Father-mother conflict & -0.11 & -0.95 & 0.10 & 0.90 \\
\hline & Family economic & 0.29 & $2.69^{* *}$ & 0.16 & 1.59 \\
\hline & pressure & & & & \\
\hline
\end{tabular}

